\title{
Low-Dose Aspirin for Primary Prevention of Cardiovascular Events in Elderly Japanese Patients with Atherosclerotic Risk Factors: Subanalysis of a Randomized Clinical Trial (JPPP-70)
}

\author{
Masahiro Sugawara ${ }^{1} \cdot$ Yoshio Goto $^{2} \cdot$ Tsutomu Yamazaki $^{3} \cdot$ Tamio Teramoto $^{4} \cdot$ Shinichi Oikawa $^{5} \cdot$ Kazuyuki Shimada $^{6}$. \\ Shinichiro Uchiyama ${ }^{7} \cdot$ Katsuyuki Ando $^{8} \cdot$ Naoki Ishizuka $^{9} \cdot$ Mitsuru Murata $^{10} \cdot$ Kenji Yokoyama $^{11}$ - Yukari Uemura ${ }^{3}$. \\ Yasuo Ikeda ${ }^{12}$. On behalf of the Japanese Primary Prevention Project (JPPP) Study Group
}

Published online: 18 December 2018

(c) The Author(s) 2018

\begin{abstract}
Introduction This post hoc subanalysis of the randomized Japanese Primary Prevention Project investigated whether oncedaily low-dose aspirin versus no aspirin reduced the risk of cardiovascular events (CVEs) in patients aged $\geq 70$ years with atherosclerotic risk factors.

Methods Patients aged $<70$ years (young-old) or $\geq 70$ years (old) with hypertension, dyslipidemia, or diabetes participated between 2005 and 2007. Patients were randomized 1:1 to receive $100 \mathrm{mg}$ enteric-coated aspirin once daily or no aspirin plus standard of care. The primary outcome was a composite of death from cardiovascular causes plus nonfatal stroke and nonfatal myocardial infarction. The secondary outcome was a composite of the primary outcome plus transient ischemic attack, angina pectoris, and arteriosclerotic disease requiring medical or surgical intervention. Old $(n=7971)$ and young-old $(n=6493)$ patients were followed up for a median 5.02 years.

Results Aspirin did not reduce the risk of primary (hazard ratio [HR] 0.92 [95\% confidence interval $\{\mathrm{CI}\}$ 0.74-1.16]; $P=0.50)$ or secondary $(0.85[0.70-1.04] ; P=0.11)$ outcomes in patients aged $\geq 70$ years. In old men with high-density lipoprotein $<40 \mathrm{mg} / \mathrm{dL}$, treatment with low-dose aspirin was associated with a reduction in the incidence of the primary endpoint compared with the group not receiving aspirin (10/260 vs $22 / 250$; HR 0.44 [95\% CI $0.20-0.93$ ]; $P=0.03)$. This subgroup was also found to contain significant larger proportions of patients with elevated body mass index, patients with diabetes mellitus, and smokers $(P<0.001)$. Old patients also showed differences in bleeding outcomes. Serious extracranial hemorrhage requiring transfusion or hospitalization occurred significantly more frequently in the aspirin-treated group than in the non-aspirin-treated group (35 [0.88\%] vs 18 [0.45\%]; HR 1.96 [1.11-3.46]; $P=0.020$ ). Gastrointestinal hemorrhage occurred significantly more frequently in the aspirin-treated group than the non-aspirin-treated group (63 [1.58\%] vs 18 [0.45\%]; relative risk [RR] 3.5 [2.08-5.90]; $P<0.0001$ ). Cerebral hemorrhage (intracranial hemorrhage) tended to occur more frequently in the aspirin-treated group than the non-aspirin-treated group (22 [0.55\%] vs 11 [0.28\%]; RR 2.01 [0.97-4.14]; $P=0.058)$. Cerebral hemorrhage occurred significantly more frequently in old patients than in young-old patients (33 [0.41\%] vs 10 [0.15\%]; HR 2.7 [1.34-5.53]; $P=0.0055)$. Gastrointestinal hemorrhage occurred in a slightly higher proportion of old patients compared with young-old patients (81 [1.02\%] vs 53 [0.82\%]; RR $1.2[0.88-1.76] ; P=0.21$ ).

Discussion/Conclusions Aspirin did not reduce the risk of the primary or secondary outcomes in old patients. Aspirin treatment may have reduced CVEs within a high CVE risk elderly population subgroup. Aspirin treatment in such a group requires caution, because of the increased risk of intracranial hemorrhage, severe extracranial hemorrhage requiring hospitalization or transfusion, and gastrointestinal bleeding in old patients receiving aspirin therapy.
\end{abstract}

Clinical Trial Registration The study is registered at ClinicalTrials.gov [NCT00225849].

Electronic supplementary material The online version of this article (https://doi.org/10.1007/s40256-018-0313-0) contains supplementary material, which is available to authorized users.

Extended author information available on the last page of the article 


\section{Key Points}

This post hoc subanalysis of the randomized Japanese Primary Prevention Project [NCT00225849] investigated the effect of once-daily low-dose aspirin versus no aspirin on cardiovascular event (CVE) risk in patients aged $\geq 70$ years with atherosclerotic risk factors.

The primary outcome was death from cardiovascular causes plus nonfatal stroke and nonfatal myocardial infarction. The secondary outcome was a composite of the primary outcome plus transient ischemic attack, angina pectoris, and arteriosclerotic disease requiring medical or surgical intervention.

Once-daily low-dose aspirin did not reduce the risk of CVEs in Japanese patients aged $\geq 70$ years with atherosclerotic risk factors.

\section{Introduction}

Cardiovascular disease is the leading cause of death, accounting for $31 \%$ of deaths worldwide in 2012 [1]. Of the estimated 17.5 million cardiovascular-related deaths in 2012, the most common causes were coronary heart disease $(42.3 \%)$ and stroke (38.3\%) [1]. The number of deaths from cardiovascular diseases has been predicted to rise to over 22 million per year by 2030 [2]. In Japan, the risk of cardiovascular mortality per 1000 person-years increases from 10.81 in men and 6.97 in women aged 60-69 years to 37.61 in men and 31.33 in women aged 70 years and older (data from 1980-1999) [3]. Japan has an aging population, and onethird of the inhabitants are predicted to be aged $\geq 65$ years by 2030 [4]. Additionally, the prevalence of risk factors for cardiovascular disease, such as obesity, glucose intolerance, and hypercholesterolemia, is increasing in this group [5], meaning that prevention of cardiovascular events (CVEs) in the elderly is a public health issue in Japan.

Treatment with low-dose aspirin for the primary prevention of cardiovascular disease has been widely investigated. However, the results of studies have been conflicting and, as a result, guidelines on the use of aspirin for primary prevention vary substantially [6]. In a meta-analysis of six primary prevention trials, the Antithrombotic Trialists' Collaboration (ATTC) found that low-dose aspirin reduced the occurrence of nonfatal myocardial infarction (MI) by $20 \%$ and that of serious vascular events by $12 \%$ [7]. More recent meta-analyses have shown that although aspirin may significantly reduce the risk of a first MI, its role in the prevention of stroke or cardiovascular-related death is unclear [6]. Furthermore, low-dose aspirin has been shown to increase the risk of gastrointestinal bleeding (GIB) and hemorrhagic stroke in both real-world and controlled-trial settings [7, 8]. However, in patients aged $\geq 70$ years, the US Preventive Services Task Force (USPSTF) has recently reported that there is currently insufficient evidence to assess the balance of benefit versus harm of using aspirin for the primary prevention of cardiovascular disease, and additional evidence in this age group is needed [9]. However, recent Japanese studies, such as the Japanese Primary Prevention Project (JPPP) [10] and Japanese Primary Prevention of Atherosclerosis with Aspirin for Diabetes (JPAD) [11] trials, did not demonstrate the overall merit of low-dose aspirin for primary prevention. Ten-year follow-up of JPAD subjects also indicated similar results [12].

The JPPP investigated the role of aspirin in the primary prevention of cardiovascular disease in more than 14,000 Japanese patients aged $\geq 60$ years who had any combination of risk factors for atherosclerosis including hypertension, dyslipidemia, and/or diabetes mellitus [10]. The JPPP found that although daily, low-dose aspirin significantly reduced the incidence of nonfatal MI and transient ischemic attack, it did not reduce the incidence of the composite outcome of cardiovascular death, nonfatal stroke, and nonfatal MI; however, event rates in the study were low and the study was terminated early owing to likely futility [10]. While event rates between patients aged $\geq 70$ years versus those aged $<70$ years were similar, results of regression analyses suggested that the risk of reaching the primary endpoint- - a composite of death from CVEs, nonfatal stroke, and nonfatal MI-was higher in patients aged $\geq 70$ years (parameter estimate 0.92; hazard ratio [HR] 2.51 [95\% confidence interval \{CI $2.00-3.14] ; P<0.001)$ [10].

This study (JPPP-70) was a post hoc subanalysis of the original JPPP study to determine whether low-dose aspirin reduced the risk of CVEs versus no aspirin, in Japanese patients aged $\geq 70$ years with atherosclerotic risk factors.

\section{Methods}

\subsection{Patient Selection}

The JPPP was a multicenter, randomized, open-label, parallel-group clinical study. The study is registered at ClinicalTrials.gov [NCT00225849], and full details of the methods and study design have been published previously $[10,13]$. Briefly, patients were eligible for the JPPP if they met the Japanese criteria for hypertension (2006 guidelines), dyslipidemia (2002), or diabetes (2004; full definitions have been provided by Ikeda and colleagues [10]) [14-16]. Patients with a medical history of coronary artery disease, cerebrovascular disease, atherosclerotic disease 
requiring medical or surgical intervention, atrial fibrillation, peptic ulcer, or bleeding disorders or abnormalities (e.g., clotting factor deficiencies) were excluded. Patients were excluded if they were receiving antiplatelet therapy, anticoagulants, or nonsteroidal anti-inflammatory drugs, or if they had previously had an adverse reaction to aspirin or salicylic acid. Patients were recruited by primary care physicians at 1007 clinics in Japan between March 2005 and June 2007.

For the purposes of this subanalysis, patients were stratified into two groups: those aged $\geq 70$ years and those aged $<70$ years.

\subsection{Study Design}

Throughout the study, all patients received standard of care treatment to control hypertension, dyslipidemia, or diabetes in accordance with Japanese guidelines [14-16]. Patients were randomized 1:1 to receive either a $100-\mathrm{mg}$ tablet of enteric-coated aspirin or no aspirin following a screening visit. A central computerized system was used to generate the random allocation sequence, and treatment allocations were disseminated to study investigators via the study website or by fax. Disease outcomes, adverse events, self-reported treatment adherence, blood pressure, serum lipid levels, blood glucose levels, smoking status, and body weight were assessed at each visit. Patients were followed up until withdrawal of consent or death. If the cause of death was unclear, the patient's death certificate was obtained with the permission of the Japanese government. The causes of all deaths were verified by the end of April 2014. Members of the study committees and details of investigator locations are available in the online supplementary material. Informed consent was obtained from each patient, and the study protocol conforms to the ethical guidelines of the 1975 Declaration of Helsinki as reflected in a priori approval by the human research committee of the Japan Physicians Association.

\subsection{Study Endpoints}

The primary endpoint was a composite of death from cardiovascular causes (MI, stroke, and other cardiovascular causes), nonfatal stroke (ischemic or hemorrhagic, including undefined cerebrovascular events), and nonfatal MI. The secondary endpoint was a composite of the primary endpoint plus transient ischemic attack, angina pectoris, and arteriosclerotic disease requiring medical or surgical intervention.

\subsection{Statistical Methods}

The statistical analyses and sample size calculations used in the main JPPP have been described previously $[10,11]$. For this subanalysis, the test hypothesis was that treatment with aspirin reduces the incidence of the primary or secondary endpoints in study participants aged $\geq 70$ years with a high risk of a CVE compared with patients aged $<70$ years. The test hypothesis was confirmed by rejecting the null hypothesis that aspirin does not have a preventive effect on cardiovascular outcome in patients aged $\geq 70$ years with or without additional cardiovascular risk factors compared with those aged $<70$ years. Between-group differences in the primary endpoint were assessed using the stratified logrank test, with stratification for underlying disease characteristics (hypertension, dyslipidemia, or diabetes mellitus) with a two-sided significance level of $\alpha=0.05$. HRs were calculated using the Cox proportional hazards model. For exploratory analyses, patients were further stratified according to known cardiovascular risk factors (sex, systolic blood pressure, high-density lipoprotein [HDL], body mass index [BMI], smoking status, and diabetes mellitus status). Twosided stratified log-rank tests and Cox proportional hazards models were performed as above.

\section{Results}

\subsection{Patients}

Of the 14,464 patients who completed the main JPPP study, 7971 (3118 men [39.1\%] and 4853 women [60.9\%]) were aged $\geq 70$ years (Fig. 1). These patients were included in this subanalysis investigating the efficacy of aspirin in the primary prevention of CVEs in patients aged $\geq 70$ years compared with patients aged $<70$ years (JPPP-70). Patients were randomized between March 2005 and June 2007, and the study was terminated following interim analysis in May 2011, owing to likely futility. The median follow-up time for this cohort was 5.02 years.

Compared with patients aged $<70$ years, patients aged $\geq 70$ years were more likely to be female, have hypertension, and have lower levels of triglycerides and total cholesterol levels (Table 1). Patients in the older age group were also less likely to be overweight, smoke, or have a family history of cardiovascular disease than younger patients. Among older patients, baseline characteristics were similar in the treatment and control groups, with the exception of glycated hemoglobin (HbA1c) levels, which were significantly higher in the control group (Table 1); the frequency of non-adherence in the intervention group (i.e., patients who did not take aspirin) was $28.0 \%(n=1118)$, and the frequency of contamination of the control group (i.e., by patients who took 


\begin{tabular}{|c|c|c|c|}
\hline $\begin{array}{l}7323 \text { patients randomized to } \\
\text { receive enteric-coated aspirin } \\
(100 \mathrm{mg} / \text { day })\end{array}$ & \multicolumn{2}{|c|}{$\begin{array}{c}14658 \text { patients } \\
\text { randomized }\end{array}$} & $\begin{array}{l}7335 \text { patients randomized to } \\
\text { receive no aspirin } \\
\text { (usual care) }\end{array}$ \\
\hline$\downarrow$ & & & $\downarrow$ \\
\hline \multirow[t]{2}{*}{$\begin{array}{l}4051 \text { patients aged } 70 \text { years or } \\
\text { older } \\
\text { - } 3974 \text { received intervention as } \\
\text { assigned } \\
\text { - } 77 \text { did not receive assigned } \\
\text { intervention } \\
-4 \text { Withdrawal of consent } \\
-28 \text { refusal of treatment } \\
-3 \text { Entry criteria not met } \\
-27 \text { no visiting } \\
-8 \text { occurrence of event or AE } \\
-1 \text { failure of allocation } \\
-6 \text { others }\end{array}$} & \multirow{2}{*}{$\begin{array}{l}3272 \text { patients younger than } 70 \\
\text { years } \\
\text { - } 3216 \text { received intervention as } \\
\text { assigned } \\
56 \text { did not receive assigned } \\
\text { intervention } \\
-1 \text { Withdrawal of consent } \\
-18 \text { refusal of treatment } \\
-3 \text { Entry criteria not met } \\
-22 \text { no visiting } \\
-4 \text { occurrence of event or AE } \\
-1 \text { failure of allocation } \\
-7 \text { others }\end{array}$} & $\begin{array}{l}4048 \text { patients aged } 70 \text { years } \\
\text { or older } \\
\text { - } 4043 \text { received intervention } \\
\text { as assigned } \\
\text { - } 5 \text { did not receive assigned } \\
\text { intervention } \\
-2 \text { failure of allocation } \\
-3 \text { request of treatment }\end{array}$ & $\begin{array}{l}3287 \text { patients younger than } \\
70 \text { years } \\
\text { - } 3287 \text { received intervention } \\
\text { as assigned }\end{array}$ \\
\hline & & & \\
\hline$\downarrow$ & $\downarrow$ & & $\downarrow$ \\
\hline $\begin{array}{l}3986 \text { patients included in } \\
\text { analysis } \\
65 \text { patients not included in } \\
\text { analysis } \\
\text { - } 39 \text { Protocol violation, delay } \\
\text { in treatment, or unreported } \\
\text { data } \\
\text { - } 14 \text { Entry criteria not met } \\
\text { - } 8 \text { Withdrawal of consent } \\
\text { - } 4 \text { Clinic or investigator } \\
\text { circumstances }{ }^{c}\end{array}$ & $\begin{array}{l}\mathbf{3 2 3 4} \text { patients included in } \\
\text { analysis } \\
\mathbf{3 8} \text { patients not included in } \\
\text { analysis } \\
\text { - } 16 \text { Protocol violation, delay } \\
\text { in treatment, or unreported } \\
\text { data }^{\mathrm{d}} \\
\text { - } 16 \text { Entry criteria not met } \\
\text { - } 6 \text { Withdrawal of consent } \\
\text { - } 0 \text { Clinic or investigator } \\
\text { circumstances }^{\mathrm{c}}\end{array}$ & $\begin{array}{l}3985 \text { patients included in } \\
\text { analysis } \\
63 \text { patients not included in } \\
\text { analysis } \\
\text { - } 38 \text { Protocol violation, delay } \\
\text { in treatment, or unreported } \\
\text { data }^{\mathrm{a}} \\
\text { - } 19 \text { Entry criteria not met }{ }^{\mathrm{b}} \\
\text { - } 1 \text { Withdrawal of consent } \\
\text { - } 5 \text { Clinic or investigator } \\
\text { circumstances }^{c}\end{array}$ & $\begin{array}{l}3259 \text { patients included in } \\
\text { analysis } \\
28 \text { patients not included in } \\
\text { analysis } \\
\text { - } 21 \text { Protocol violation, delay } \\
\text { in treatment, or unreported } \\
\text { data }^{d} \\
\text { - } 6 \text { Entry criteria not met } \\
\text { - } 0 \text { Withdrawal of consent } \\
\text { - } 1 \text { Clinic or investigator } \\
\text { circumstances }^{c}\end{array}$ \\
\hline
\end{tabular}

Fig. 1 Flow of patients through the Japanese Primary Prevention Project-70 (JPPP-70) subanalysis. $A E$ adverse event. Age 70 years

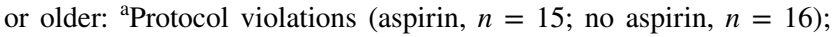
delay in start of treatment (aspirin, $n=10$; no aspirin, $n=10$ ); unreported data by investigators in the clinics (aspirin, $n=14$; no aspirin, $n=12$ ). ${ }^{b}$ Reasons for not meeting inclusion criteria were serious blood abnormalities (aspirin, $n=1$ ), history of prohibited drugs (aspirin, $n=4$; no aspirin, $n=13$ ), cerebrovascular disease (aspirin, $n=4$; no aspirin, $n=6$ ), atrial fibrillation (aspirin, $n=1$ ), hypersensitivity to aspirin (aspirin, $n=3$ ), or atherosclerotic disease (aspirin,

at least one dose of aspirin) was 5.2\% ( $n=206)$. The number and proportion of non-adherent patients in the aspirin group were calculated from patients stating that they were "not taking aspirin" as responses for current medication at any of the once-annual follow-up examinations. Adverse events $(n=517)$ represented the major reason for stopping aspirin treatment, with gastrointestinal discomfort as the most frequently occurring adverse event. Patients in the control group who took aspirin on at least one occasion represented contamination of this group. Of the 206 patients so classified, 104 took aspirin in association with occurrence of an event, and another 19 took aspirin on their own volition. $n=1) .{ }^{\mathrm{c}}$ Clinic or investigator circumstances were closure of clinic and investigator death. Younger than 70 years: ${ }^{\mathrm{d}}$ Protocol violations (aspirin, $n=4$; no aspirin, $n=6$ ); delay in start of treatment (no aspirin, $n=5$ ); unreported data by investigators in the clinics (aspirin, $n=12$; no aspirin, $n=10$ ). ${ }^{\text {e}}$ Reasons for not meeting inclusion criteria were serious blood abnormalities (aspirin, $n=1$ ), history of prohibited drugs (aspirin, $n=8$; no aspirin, $n=5$ ), cerebrovascular disease (aspirin, $n=2$; no aspirin, $n=1$ ), atrial fibrillation (aspirin, $n=2$ ), peptic ulcer (aspirin, $n=2$ ), or long-term use of nonsteroidal anti-inflammatory drugs (aspirin, $n=1$ )

\subsection{Effectiveness}

\subsubsection{Primary Outcomes}

The rates of primary events per 1000 person-years were as follows (Table 2): total cohort 5.88 (men 7.48; women 4.73); patients aged $\geq 70$ years 7.93 (men 10.01; women 6.62); and patients aged $<70$ years 3.41 (men 4.90 ; women 2.15 ). In the older group, event rates per 1000 person-years were 7.60 in patients receiving aspirin and 8.25 in patients not receiving aspirin.

More primary endpoint CVEs occurred in patients aged $\geq 70$ years than in those aged $<70$ years ( 295 vs 105 events) (Table 3). Both fatal and nonfatal primary endpoint CVEs occurred more frequently in the older age group (92 vs 20 and 203 vs 85 events, respectively). Overall, in patients 
Table 1 Baseline characteristics (modified intention-to-treat population)

\begin{tabular}{|c|c|c|c|c|c|c|}
\hline \multirow[t]{3}{*}{ Characteristic } & \multicolumn{6}{|l|}{ Age } \\
\hline & \multirow[t]{2}{*}{$\geq 70$ years $(n=7971)$} & \multirow[t]{2}{*}{$<70$ years $(n=6493)$} & \multirow[t]{2}{*}{$P$ value } & \multicolumn{3}{|l|}{$\geq 70$ years $(n=7971)$} \\
\hline & & & & Aspirin $(n=3986)$ & No aspirin $(n=3985)$ & $P$ value \\
\hline \multicolumn{7}{|l|}{ Patient demographics } \\
\hline Age, mean (SD), years & $75.2(3.9)$ & $64.8(2.8)$ & $<0.0001$ & $75.2(3.9)$ & $75.2(3.9)$ & 0.822 \\
\hline Male, $n(\%)$ & $3118(39.1)$ & $3005(46.3)$ & $<0.0001$ & $1555(39.0)$ & $1563(39.2)$ & 0.847 \\
\hline $\begin{array}{l}\text { Waist circumference, mean } \\
\text { (SD), cm }\end{array}$ & $84.9(10.2)$ & $85.0(9.7)$ & 0.764 & $84.9(10.1)$ & $84.9(10.1)$ & 0.715 \\
\hline Weight, mean (SD), kg & $56.6(9.9)$ & $61.2(10.4)$ & $<0.0001$ & $56.7(9.9)$ & $56.6(9.9)$ & 0.668 \\
\hline BMI, mean (SD), $\mathrm{kg} / \mathrm{m}^{2}$ & $23.9(3.4)$ & $24.5(3.4)$ & $<0.0001$ & $23.9(3.4)$ & $23.9(3.4)$ & 0.787 \\
\hline $\mathrm{BMI} \geq 25 \mathrm{~kg} / \mathrm{m}^{2}, n(\%)$ & $2653(33.3)$ & $2593(39.9)$ & $<0.0001$ & $1326(33.3)$ & $1327(33.3)$ & 0.975 \\
\hline \multicolumn{7}{|c|}{ Risk factors for vascular events, $n(\%)$} \\
\hline HT & $6916(86.8)$ & $5362(82.6)$ & $<0.0001$ & $3459(86.8)$ & $3457(86.8)$ & 0.970 \\
\hline DL & $5565(69.8)$ & $4833(74.4)$ & $<0.0001$ & $2791(70.0)$ & $2774(69.6)$ & 0.691 \\
\hline $\mathrm{DM}$ & $2597(32.6)$ & $2306(35.5)$ & 0.0002 & $1297(32.5)$ & $1300(32.6)$ & 0.937 \\
\hline HT and DL & $4664(58.5)$ & $3876(59.7)$ & 0.150 & $2340(58.7)$ & $2324(58.3)$ & 0.726 \\
\hline DL and DM & $1853(23.2)$ & $1739(26.8)$ & $<0.0001$ & $928(23.3)$ & $925(23.2)$ & 0.942 \\
\hline HT and DM & $2117(26.6)$ & $1754(27.0)$ & 0.539 & $1058(26.5)$ & 1059 (26.6) & 0.974 \\
\hline HT, DL, and DM & $1527(19.2)$ & $1361(21.0)$ & 0.0069 & $765(19.2)$ & $762(19.1)$ & 0.936 \\
\hline \multicolumn{7}{|l|}{ Blood pressure, $\mathrm{mmHg}$} \\
\hline Systolic & $137.7(15.3)$ & $136.5(16.1)$ & $<0.0001$ & $137.6(15.3)$ & $137.9(15.4)$ & 0.390 \\
\hline Diastolic & $76.3(10.2)$ & $79.4(10.3)$ & $<0.0001$ & $76.1(10.2)$ & $76.4(10.1)$ & 0.223 \\
\hline Currently smoking, $n(\%)$ & $784(9.8)$ & $1109(17.1)$ & $<0.0001$ & $399(10.0)$ & $385(9.7)$ & 0.601 \\
\hline \multicolumn{7}{|c|}{ Family history of premature CV disease, $n(\%)$} \\
\hline No & $4497(56.4)$ & $3647(56.2)$ & $<0.0001$ & $2244(56.3)$ & $2253(56.5)$ & 0.975 \\
\hline Yes & $2080(26.1)$ & $1883(29.0)$ & - & $1042(26.1)$ & $1038(26.0)$ & - \\
\hline Unknown & $1394(17.5)$ & $963(14.8)$ & - & $700(17.6)$ & $694(17.4)$ & - \\
\hline \multicolumn{7}{|l|}{ Laboratory values, mean (SD) } \\
\hline \multicolumn{7}{|l|}{ Cholesterol, mg/dL } \\
\hline Total & $200.7(32.4)$ & $206.4(32.9)$ & $<0.0001$ & $200.4(32.5)$ & $201.0(32.3)$ & 0.420 \\
\hline Low-density lipoprotein ${ }^{\mathrm{a}}$ & $118.3(29.8)$ & $121.0(31.1)$ & $<0.0001$ & $118.1(29.7)$ & $118.5(29.9)$ & 0.551 \\
\hline High-density lipoprotein & $57.5(15.3)$ & $58.6(16.3)$ & $<0.0001$ & $57.3(15.4)$ & $57.8(15.1)$ & 0.162 \\
\hline Triglycerides & $126.9(68.6)$ & $138.1(83.8)$ & $<0.0001$ & $127.8(69.6)$ & $125.9(67.6)$ & 0.209 \\
\hline Fasting blood glucose, mg/dL & $106.8(29.5)$ & $108.9(34.0)$ & $<0.0001$ & $107.1(30.2)$ & $106.4(28.7)$ & 0.312 \\
\hline $\mathrm{HbA} 1 \mathrm{c}, \%^{\mathrm{b}}$ & $5.6(0.9)$ & $5.7(1.1)$ & $<0.0001$ & $5.6(0.9)$ & $5.7(1.1)$ & $<0.0001$ \\
\hline
\end{tabular}

SI conversion factors: To convert total, low-density lipoprotein, and high-density lipoprotein cholesterol to mmol/L, multiply by 0.0259 ; to convert triglycerides to $\mathrm{mmol} / \mathrm{L}$, multiply by 0.0113 ; to convert glucose to $\mathrm{mmol} / \mathrm{L}$, multiply by 0.0555

$B M I$ body mass index (calculated as weight in $\mathrm{kg}$ divided by height in $\mathrm{m}^{2}$ ), $C V$ cardiovascular, $D L$ dyslipidemia, $D M$ diabetes mellitus, $H b A l c$ glycated hemoglobin, $H T$ hypertension, $S D$ standard deviation

${ }^{a}$ Calculated based on the Friedewald formula and direct measurements

${ }^{b}$ National Glycohemoglobin Standardization Program method

aged $<70$ years, the event rate was $1.6 \%$ in both the group receiving aspirin and the group receiving no aspirin. In patients aged $\geq 70$ years, the event rate was $3.5 \%$ in the group receiving aspirin and $3.9 \%$ in the group receiving no aspirin.

Aspirin was not associated with a reduction in risk of the primary outcome measure versus no aspirin in patients aged $\geq 70$ years (HR 0.92 [95\% CI $0.74-1.16$ ]; $P=0.50$ ). Comparisons of the aspirin and non-aspirin-treated populations in this study were expected to be possible because they were both subgroups of randomized and comparable populations. However, data were analyzed with a Cox proportional hazards model comprising the characteristics in Table 1 (age, sex, BMI, hypertension, dyslipidemia, diabetes mellitus, smoking status, family history of cardiovascular disease, HDL, low-density lipoprotein [LDL], and triglycerides, fasting blood glucose, and $\mathrm{HbA} 1 \mathrm{c}$ 
Table 2 Primary event rates (modified intention-to-treat population)

\begin{tabular}{lcc}
\hline Group & Primary events & $\begin{array}{l}\text { Event rate/1000 } \\
\text { person-years }\end{array}$ \\
\hline Men & 213 & 7.48 \\
Women & 187 & 4.73 \\
Total (both sexes) & 400 & 5.88 \\
$\geq 70$ years & & \\
Men & 144 & 10.01 \\
Women & 151 & 6.62 \\
Total (both sexes) & 295 & 7.93 \\
Aspirin & 141 & 7.60 \\
Non-aspirin & 154 & 8.25 \\
$<70$ years & & \\
Men & 69 & 4.90 \\
Women & 36 & 2.15 \\
Total (both sexes) & 105 & 3.41 \\
\hline
\end{tabular}

levels). This analysis yielded an adjusted HR of 0.92 (95\% CI $0.73-1.16 ; P=0.49$ ), which was almost unchanged from that in crude analysis. A similar result-an HR of 0.93 (95\% CI $0.67-1.29 ; P=0.68$ - — was obtained in sensitivity analysis in which groups were compared using propensity score with an inverse probability weighted method.

While the number of fatal CVEs was the same $(n=46)$ in the groups aged $\geq 70$ years receiving aspirin or no aspirin, the number of fatal cerebral infarction or subarachnoid hemorrhage events was lower in the group receiving aspirin (one vs five cases and one vs four cases, respectively). However, the number of "other" fatal CVEs was higher in the group receiving aspirin ( 35 cases vs 26 cases). In patients aged $<70$ years, the number of each type of fatal event was broadly the same in the group receiving aspirin and the group receiving no aspirin. There was a suggestion that aspirin was associated with a reduction in the number of nonfatal MIs compared with no aspirin (13 vs 29 events) in patients aged $\geq 70$ years; however, more nonfatal intracranial hemorrhage (ICH) occurred in aspirin-treated patients (17 vs six events). Survival curves present the differences in primary and secondary outcomes between aspirin-treated and non-aspirin-treated patients (Online Resource 1, see the electronic supplementary material).

In patients aged $\geq 70$ years, analysis of subgroups based on common risk factors for CVEs suggested that having an HDL level of $<40 \mathrm{mg} / \mathrm{dL}$ (HR 1.72 [95\% CI 1.30-2.27]; $P=0.0001$ ), smoking (HR 1.66 [95\% CI 1.30-2.13]; $P<0.0001$ ), or having diabetes mellitus (HR 1.63 [95\% CI 1.33-1.99]; $P<0.0001)$ was associated with occurrence of the primary outcome measure (Online Resource 2). However, there was no difference in the incidence of primary outcome events between patients receiving aspirin and those not receiving aspirin in the older patient subgroup
(Online Resource 3 ). In men aged $\geq 70$ years with HDL levels of $<40 \mathrm{mg} / \mathrm{dL}$, treatment with low-dose aspirin was associated with a reduction in the incidence of the primary endpoint compared with the group not receiving aspirin ( $10 / 260$ vs $22 / 250$; HR 0.44 [95\% CI $0.20-0.93$ ]; $P=0.03$ ) (Table 4). This cohort comprised 3.5\% of the original JPPP study population.

Predicted 10-year atherosclerotic cardiovascular disease (ASCVD) risks are presented in Online Resource 4. Aspirin tended to be more effective in a high-risk population (there was no significant interaction). Although the number of events was small and the difference was not significant, the HR for aspirin-treated patients versus non-aspirin-treated patients was low, at 0.651 , among patients aged $\geq 70$ years with an ASCVD risk of $\geq 15 \%$. Furthermore, the event rates in all patients with an ASCVD risk of $\geq 15 \%$ were high, at $11.2 \%$ for aspirin-treated patients and $16.7 \%$ for non-aspirin-treated patients. The ASCVD risk $\geq 15 \%$ subgroup also showed a small number of primary endpoint events in patients aged $<70$ years, in five cases against 70 cases in patients aged $\geq 70$ years.

The mean 10-year ASCVD risk for men aged $\geq 70$ years with $\mathrm{HDL}<40 \mathrm{mg} / \mathrm{dL}$ was $14.6 \%$, signifying a high-risk population (for ASCVD). In an evaluation of background characteristics, this subgroup was also found to contain significant larger proportions of patients with elevated BMI, dyslipidemia, and diabetes mellitus, and those who were smokers $(P<0.001)$ (Online Resource 5).

\subsubsection{Secondary Outcomes}

Similar to the primary endpoint, the number of secondary endpoint events was higher in patients aged $\geq 70$ years than in those aged $<70$ years (1285 vs 612 events) (Table 3 ). Overall, aspirin treatment was not associated with a reduced risk of atherosclerotic events or CVEs in patients aged $\geq 70$ years compared with no aspirin (HR 0.85 [95\% CI $0.70-1.04] ; P=0.11)$. However, in patients aged $\geq 70$ years, there were fewer cases of nonfatal MI (13 vs 29 events), angina pectoris (20 vs 30 events), and arteriosclerotic disease requiring treatment (36 vs 53 events) in the group that received aspirin compared with those who received no aspirin. In both age groups, aspirin was associated with a reduced risk of transient ischemic attack $(<70$ years 4 vs 13 events; $\geq 70$ years 15 vs 21 events), but with an increased risk of serious extracranial hemorrhage $(\mathrm{ECH})$ requiring transfusion or hospitalization $(<70$ years 27 vs 16 events; $\geq 70$ years 35 vs 18 events).

In patients aged $\geq 70$ years, having an HDL level of $<40 \mathrm{mg} / \mathrm{dL}$ (HR 1.62 [95\% CI 1.29-2.03]; $P<0.0001$ ), smoking (HR 1.50 [95\% CI $1.22-1.85] ; P=0.0001$ ), or having diabetes mellitus (HR 1.58 [95\% CI 1.35-1.87]; $P<0.0001)$ was also associated with the occurrence of 
Table 3 Primary and secondary endpoints for patients aged 70 years and older and patients aged $<70$ years (modified intention-to-treat population)

\begin{tabular}{|c|c|c|c|c|c|}
\hline \multirow[t]{3}{*}{ Endpoint } & \multicolumn{4}{|l|}{ No. of events } & \multirow{3}{*}{$\begin{array}{l}\text { Interaction } \\
P \text { value }\end{array}$} \\
\hline & \multicolumn{2}{|l|}{$<70$ years } & \multicolumn{2}{|l|}{$\geq 70$ years } & \\
\hline & Aspirin $(n=3234)$ & No aspirin $(n=3259)$ & Aspirin $(n=3986)$ & No aspirin $(n=3985)$ & \\
\hline \multicolumn{6}{|l|}{ Primary endpoint } \\
\hline Fatal:nonfatal events & $10: 42$ & $10: 43$ & $46: 95$ & $46: 108$ & - \\
\hline Cerebral infarction & $1: 24$ & $2: 27$ & $1: 59$ & $5: 67$ & - \\
\hline Intracranial hemorrhage & $0: 6$ & $0: 4$ & $5: 17$ & $5: 6$ & - \\
\hline Subarachnoid hemorrhage & $1: 4$ & 0:0 & $1: 4$ & $4: 4$ & - \\
\hline Myocardial infarction & $3: 7$ & $3: 9$ & $4: 13$ & $6: 29$ & - \\
\hline $\begin{array}{l}\text { Other (fatal)/undefined (nonfatal) cardio- } \\
\text { vascular events }\end{array}$ & $5: 1$ & $5: 3$ & $35: 2$ & $26: 2$ & - \\
\hline Total primary events & 52 & 53 & 141 & 154 & 0.745 \\
\hline \multicolumn{6}{|l|}{ Secondary endpoint } \\
\hline $\begin{array}{l}\text { Any atherosclerotic or cardiovascular } \\
\text { event }^{\mathrm{a}}\end{array}$ & 96 & 102 & 184 & 217 & 0.524 \\
\hline Death from cardiovascular disease & 10 & 10 & 48 & 47 & 0.968 \\
\hline $\begin{array}{l}\text { Death from causes other than cardiovas- } \\
\text { cular disease }\end{array}$ & 66 & 62 & 173 & 184 & 0.545 \\
\hline $\begin{array}{l}\text { Nonfatal cerebrovascular disease } \\
\text { (ischemic or hemorrhagic) }\end{array}$ & 35 & 34 & 82 & 80 & 0.970 \\
\hline Nonfatal myocardial infarction & 7 & 9 & 13 & 29 & 0.362 \\
\hline Transient ischemic attack & 4 & 13 & 15 & 21 & 0.205 \\
\hline Angina pectoris & 26 & 24 & 20 & 30 & 0.225 \\
\hline $\begin{array}{l}\text { Arteriosclerotic diseases requiring sur- } \\
\text { gery or intervention }\end{array}$ & 39 & 32 & 36 & 53 & 0.662 \\
\hline $\begin{array}{l}\text { Severe extracranial hemorrhage requiring } \\
\text { transfusion or hospitalization }\end{array}$ & 27 & 16 & 35 & 18 & 0.748 \\
\hline Total secondary events (first event) ${ }^{\mathrm{c}}$ & $310(181)$ & $302(172)$ & $606(370)$ & $679(399)$ & $-(0.300)$ \\
\hline
\end{tabular}

${ }^{a}$ Death from cardiovascular causes, nonfatal stroke (ischemic or hemorrhagic), nonfatal myocardial infarction, transient ischemic attack, angina pectoris, and arteriosclerotic disease requiring surgery or intervention

${ }^{\mathrm{b}}$ Test for heterogeneity among treatment vs control hazard ratios between patients aged $\geq 70$ years and those aged $<70$ years

${ }^{c}$ Owing to some patients experiencing multiple secondary events in this study, the test for heterogeneity was performed using data on those secondary events that occurred first in each patient

secondary endpoint events (Online Resource 1). The incidence of secondary outcome events in older patients was similar with and without aspirin treatment (Online Resource 2).

\subsection{Safety and Tolerability}

GIB occurred in 63 patients $(1.6 \%)$ aged $\geq 70$ years who received aspirin, compared with 18 patients $(0.5 \%)$ in the same age group who did not receive aspirin $(P<0.0001)$ (Table 5). Patients aged $\geq 70$ years treated with aspirin also seemed to be at increased risk of developing peptic ulcers, heartburn, stomach/abdominal pain, and stomach/abdominal discomfort compared with those not treated with aspirin. In the men aged $\geq 70$ years with HDL levels of $<40 \mathrm{mg} / \mathrm{dL}$, there were no cases of severe ECH requiring transfusion or hospital admission, such as GIB, in the aspirin-treated groups (Table 4).

Evaluations of patients aged $\geq 70$ years revealed the following. Fatal ICH occurred in two aspirin-treated and two non-aspirin-treated men, and three aspirin-treated and three non-aspirin-treated women. Nonfatal ICH occurred in four aspirin-treated and two non-aspirin-treated men, and 13 aspirin-treated and four non-aspirin-treated women. This yielded totals of 22 and 11 ICHs in aspirintreated and non-aspirin-treated patients, respectively, involving ten men and 23 women. More detailed evaluations were attempted by risk-factor subgrouping for fatal and nonfatal ICH event rates in patients aged $\geq 70$ years. As shown in Online Resource 6, event rates tended to be high within the following subgroups: female, $\mathrm{LDL}<140 \mathrm{mg} / \mathrm{dL}, \mathrm{BMI}<25$, and family CVD history. 
Table 4 Primary and secondary endpoints in men aged $\geq 70$ years with high-density lipoprotein levels $<40 \mathrm{mg} / \mathrm{dL}$ (modified intention-to-treat population)

\begin{tabular}{|c|c|c|}
\hline \multirow[t]{2}{*}{ Endpoint } & \multicolumn{2}{|l|}{ No. of events } \\
\hline & Aspirin $(n=260)$ & $\begin{array}{l}\text { No aspirin } \\
(n=250)\end{array}$ \\
\hline \multicolumn{3}{|l|}{ Primary endpoint } \\
\hline Fatal events & 2 & 5 \\
\hline Cerebral infarction & 0 & 1 \\
\hline Intracranial hemorrhage & 0 & 0 \\
\hline Subarachnoid hemorrhage & 0 & 1 \\
\hline Myocardial infarction & 0 & 1 \\
\hline Other fatal cardiovascular events & 2 & 2 \\
\hline Nonfatal events & 8 & 17 \\
\hline Cerebral infarction & 5 & 8 \\
\hline Intracranial hemorrhage & 1 & 1 \\
\hline Subarachnoid hemorrhage & 0 & 0 \\
\hline Myocardial infarction & 2 & 8 \\
\hline Undefined cerebrovascular events & 0 & 0 \\
\hline Total primary events & 10 & 22 \\
\hline \multicolumn{3}{|l|}{ Secondary endpoint } \\
\hline Any atherosclerotic or cardiovascular event & 9 & 14 \\
\hline Angina pectoris & 0 & 2 \\
\hline Arteriosclerotic diseases requiring surgery or intervention & 8 & 11 \\
\hline Severe extracranial hemorrhage requiring transfusion or hospitalization & 0 & 1 \\
\hline Total secondary events & 17 & 28 \\
\hline
\end{tabular}

Table 5 Incidence of prespecified gastrointestinal adverse events in patients aged $\geq 70$ years with cardiovascular risk factors receiving aspirin or no aspirin (randomized population)

\begin{tabular}{lllc}
\hline & $\begin{array}{l}\text { Aspirin }(n=3986) \\
n(\%)\end{array}$ & \multicolumn{2}{l}{$\begin{array}{l}\text { No aspirin }(n=3985) \\
n(\%)\end{array}$} \\
\hline Stomach/abdominal discomfort & $199(5.0)$ & $95(2.4)$ & $84(2.1)$ \\
Heartburn & $112(2.8)$ & $46(1.2)$ & 0.043 \\
Gastroduodenal ulcer & $106(2.7)$ & $72(1.8)$ & $<0.0001$ \\
Reflux esophagitis & $83(2.1)$ & $33(0.8)$ & 0.373 \\
Stomach/abdominal pain & $78(2.0)$ & $18(0.5)$ & $<0.0001$ \\
Gastrointestinal bleeding & $63(1.6)$ & $27(0.7)$ & 0.0719 \\
Erosive gastritis & $43(1.1)$ & $25(0.6)$ & 0.100 \\
Nausea & $38(1.0)$ & $11(0.3)$ & 0.256 \\
Stomach/abdominal pressure & $17(0.4)$ & & \\
\hline
\end{tabular}

Fatal ICH occurred in six aspirin-treated patients and four non-aspirin-treated patients. Patients aged $\geq 70$ years requiring hospitalization or transfusion for serious $\mathrm{ECH}$ were also evaluated (Table 3). There were 35 such cases among aspirin-treated patients and 18 among non-aspirin-treated patients. As shown in Online Resource 6, riskfactor subgroup analyses revealed high incidences in the male, hypertension, and family CVD history subgroups. Among men aged $\geq 70$ years with $\mathrm{HDL}<40 \mathrm{mg} / \mathrm{dL}$, no fatal ICH occurred in either aspirin-treated or non-aspirintreated patients, and one case of nonfatal ICH per group occurred for both aspirin-treated and non-aspirin-treated patients. Severe ECH requiring hospitalization or transfusion occurred with low incidence, zero and one in aspirintreated patients and non-aspirin-treated patients, respectively. GIB occurred more frequently in aspirin-treated patients, at six cases $(2.3 \%)$, than in non-aspirin-treated patients, at one case $(0.4 \%)$. 
GIB occurred more frequently in patients aged $\geq 70$ years than in patients aged $<70$ years, in whom the incidence was 40 cases $(1.2 \%)$ with aspirin treatment and $13(0.4 \%)$ with no aspirin treatment.

\section{Discussion}

JPPP-70 is a subanalysis of the JPPP, investigating the efficacy of low-dose aspirin for primary prevention of cardiovascular disease in Japanese patients aged $\geq 70$ years with atherosclerotic risk factors. As in the main JPPP study [10], the findings confirmed that low-dose aspirin did not reduce the incidence of the composite outcome of cardiovascular death, nonfatal stroke, and nonfatal $\mathrm{MI}$ in patients aged $\geq 70$ years compared with those aged $<70$ years. This is in line with findings of the ATTC meta-analysis, which showed no evidence of an age interaction [17]. Stratification by common cardiovascular risk factors suggested that lowdose aspirin was associated with a reduction in the incidence of CVEs in men aged $\geq 70$ years who also had low levels of HDL ( $<40 \mathrm{mg} / \mathrm{dL}$ ). An HDL level of $<40 \mathrm{mg} / \mathrm{dL}$ is a known risk factor for cardiovascular disease; furthermore, registry data from the USA show that the prevalence of HDL levels of $<40 \mathrm{mg} / \mathrm{dL}$ is approximately three times higher in men than in women (29.5\% vs $9.7 \%$, respectively) [15]. Thus, further investigation of the role of low-dose aspirin for primary prevention of CVEs in men with an HDL level of $<40 \mathrm{mg} /$ $\mathrm{dL}$ may be warranted in Japanese and non-Japanese patients.

We previously reported that nonfatal ICH and subarachnoid hemorrhage were more common in patients who received aspirin than in those who did not receive aspirin [10]. Additionally, more recent subanalyses of the JPPP cohort have shown that aspirin did not change the rate of fatal or nonfatal stroke [18]. In JPPP-70, there was a suggestion that aspirin was associated with an increased incidence of nonfatal ICH in individuals aged $\geq 70$ years, but not in the younger age group. In contrast, aspirin may be associated with an increased incidence of subarachnoid hemorrhage in patients aged $<70$ years only. This differs from UK registry data that suggest that aspirin does not affect the risk of $\mathrm{ICH}$, but reduces the risk of subarachnoid hemorrhage; the mean ages at diagnosis in these groups were 70.8 years and 57.7 years, respectively [19].

The occurrence of GIB, peptic ulcer, and stomach/ abdominal discomfort or pain was significantly increased in patients aged $\geq 70$ years who received aspirin compared with those who did not. Indeed, it has been reported previously that low-dose aspirin is associated with an increased risk of GIB and ICH in men older than 70 years $[8,20]$. Furthermore, increasing age is an independent risk factor for major bleeding events, including ICH [21, 22]. However, in this study the proportions of aspirin-treated patients aged $\geq 70$ years who experienced these bleeding events were still low, ranging from $1.6 \%$ for GIB to $5.0 \%$ for stomach/ abdominal discomfort.

In a cohort of nearly 2000 patients with an ST segment elevation MI, nearly $70 \%$ had not been previously diagnosed with cardiovascular disease, despite the presence of known risk factors (e.g., hypertension, dyslipidemia, and diabetes mellitus) in a high proportion of them [23]. Multiple trials and meta-analyses [7, 24-27], including the original JPPP study [10], have found that aspirin reduces the risk of nonfatal MI. However, given the increased risk of bleeding adverse events, current guidelines only recommend the use of aspirin for primary prevention of cardiovascular disease in patients with risk factors for cardiovascular or cerebrovascular disease [6].

Current US guidelines propose that low-dose aspirin is suitable for patients with a 10 -year CVE risk of $10 \%$ or greater (or a 10 -year risk of $5-10 \%$ in patients with type 2 diabetes [28]) [9, 29, 30]; UK guidelines suggest that patients with a 10 -year CVE risk of $20 \%$ may be treated with low-dose aspirin; however, primary prevention of CVD is not a licensed indication for aspirin in the UK [30]. There are currently no Japanese guidelines for the use of aspirin for the primary prevention of cardiovascular disease. Our data suggest that, similar to American and European guidelines, aspirin should only be used for the primary prevention of cardiovascular disease in Japanese patients who are at high risk of experiencing a CVE.

The limitations of this subanalysis (JPPP-70) are similar to those of the original JPPP study, which was terminated early owing to unexpectedly low rates of events and likely futility [10]. In addition, the post hoc subanalyses reported here were performed on relatively small patient subgroups and are considered to be exploratory. Furthermore, decreasing adherence levels in the group receiving aspirin and the increasing uptake of aspirin in the group not randomized to receive aspirin during the 5-year trial may have confounded between-group differences [10]. In addition, due to the open-label nature of the trial, patients receiving aspirin may have been more likely to report the occurrence of adverse events than those not receiving aspirin [10]. Finally, the subgroup that appeared to benefit from aspirin treatment, namely men aged $\geq 70$ years with HDL levels of $<40 \mathrm{mg} / \mathrm{dL}$, was small, comprising just $3.5 \%$ of the original JPPP cohort; therefore, the possibility that these results occurred owing to chance cannot be ruled out. However, the mean 10-year ASCVD risk for this subgroup was $14.6 \%$, signifying a high-risk population. Background characteristics were assessed for this group, revealing significant high proportions of patients with diabetes mellitus and smokers. Low HDL, diabetes mellitus, and smoking were noted as significant risk factors for CVEs in patients aged $\geq 70$ years, 
and this subgroup was a population with each of these risk factors. Smoking is known to decrease HDL levels. In diabetes mellitus, triglycerides are increased and HDL level is decreased through the decline in insulin action. The fact that the subgroup of men aged $\geq 70$ years with low HDL levels represented a high-risk population for CVEs may explain the significant difference obtained for aspirin-treated patients. The low incidences of the ICH and severe $\mathrm{ECH}$ in this subgroup were attributed to the low proportions of patients with high HDL, patients with low LDL, and non-smokers that were more common in both groups in this study. In patients aged $\geq 70$ years, ICH occurred in 22 aspirin-treated patients and 11 non-aspirin-treated patients; severe ECH requiring hospitalization or transfusion occurred in 35 aspirin-treated patients and 18 non-aspirin-treated patients. GIB occurred in 63 aspirin-treated patients (1.6\%) and 18 non-aspirin-treated patients $(0.5 \%)$. Incidences were significantly higher in aspirin-treated patients for each of these events. Furthermore, intracranial and severe ECH increase with age at 70 years and above. In this subgroup, GIB was noted more frequently among aspirin-treated patients than in non-aspirin-treated patients (six cases, $2.3 \%$, vs one case, $0.4 \%$ ). In light of these findings, it is considered that aspirin treatment should be approached with caution in such a high-risk population. A meta-analysis of eight primary prevention trials of low-dose aspirin in patients $<70$ years showed that, whereas aspirin-treated patients have an increased risk of severe bleeding, the advantages of aspirin treatment outweigh the disadvantages in patients with a $>10 \%$ 10-year ASCVD risk [31, 32]. Recently, three primary prevention studies of aspirin treatment have been reported. The risk of major bleeding events was significantly increased with aspirin treatment-with an HR of 1.38 - in the ASPREE study in elderly patients (mainly in patients aged $\geq 70$ years; mean age 74 years) [32, 33]. Major bleeding events also occurred significantly more frequently with aspirin treatment, with a relative risk (RR) of 1.29, in the ASCEND study in patients with diabetes aged $\geq 40$ years without cardiovascular disease [34]. The risk was similarly elevated in the present study (JPPP-70), with an RR of 1.82 .

Hemorrhagic stroke requires particular consideration. However, reported incidences of hemorrhagic stroke in aspirin-treated versus non-aspirin-treated populations were $0.13 \%$ versus $0.18 \%$ during 5 years of follow-up in the ARRIVE study in patients with a CVD risk factor (men aged $\geq 55$ years and women aged $\geq 60$ years; mean age 64 years) [35], $0.3 \%$ versus $0.3 \%$ in the 7.4 -year follow-up period in the ASCEND study, and $0.5 \%$ versus $0.4 \%$ in the 4.7-year follow-up period in the ASPREE study. The incidences were lower in these three studies than in the present study (JPPP-70), in which the corresponding incidence in aspirin-treated versus non-aspirin-treated patients during the 5.2 -year follow-up period was $0.83 \%$ versus $0.58 \%$. However, this finding was considered to reflect the high mean age in this study (75 years) and the higher incidence of stroke in the Japanese population compared with that in European and North American populations.

ICHs are common in hypertension patients, and hypertension was present in 52 of the 53 patients with serious ECH in this study. Accordingly, aspirin treatment may be useful in non-hypertensive patients with a high ASCVD risk, who have a low risk of bleeding. Furthermore, among such high-risk patients, cases of advanced coronary lesions may not be common. The required therapeutic strategy involves ankle-brachial index and carotid ultrasonography, coronary computed tomography $(\mathrm{CT})$, cardiac catheterization in some cases, objective assessments of coronary lesions in individual patients, and, where necessary, coronary angioplasty and bypass surgery.

In practice, patients on aspirin-based antiplatelet therapy but not receiving standard proton pump inhibitor (PPI) therapy have higher risk of major bleeding in the long term, and this is more persistent in older patients than in younger patients in previous trials.

Routine co-prescription is recommended because $50 \%$ of the major bleeding events in patients aged 75 years or older were upper gastrointestinal in origin, and thus the projected number of those needing PPI treatment to prevent such bleeding is low $[33,34]$. It is considered that PPIs should be administered as concomitant medication for elderly patients taking low-dose aspirin to prevent GIB.

Further analyses of the JPPP cohort are in progress, including investigations into the role of aspirin in the prevention of cancer-associated deaths, in order to identify patients in Japan who may benefit most from aspirin treatment.

\section{Conclusion}

Low-dose aspirin did not significantly reduce the risk of cardiovascular death, nonfatal stroke, or nonfatal MI in Japanese patients aged $\geq 70$ years. However, low-dose aspirin may reduce the risk of these CVEs in Japanese men aged $\geq 70$ years who also have HDL levels of $<40 \mathrm{mg} / \mathrm{dL}$. This subgroup included high proportions of patients with diabetes mellitus and smokers and had a mean 10-year ASCVD risk of $14.6 \%$, signifying that this was a high-risk population. In patients aged $\geq 70$ years, aspirin treatment yielded significantly higher incidences of serious $\mathrm{ECH}$ requiring transfusion or hospitalization and gastrointestinal hemorrhage, and tended to yield a higher incidence of cerebral hemorrhage. Cerebral hemorrhage was significantly more frequent and gastrointestinal hemorrhage was proportionally slightly more frequent in patients aged $\geq 70$ years 
than in those aged $<70$ years. Patients aged $\geq 70$ years had a greater incidence of CVEs than those aged $<70$ years, and were considered to represent a population with a higher risk of hemorrhage. The risk factors for ICH and serious ECH differed from those for CVEs.

In light of these findings, aspirin treatment for such highrisk population should be approached with caution. We consider it important to perform ankle-brachial index testing, carotid ultrasonography, coronary computed tomography angiography (CCTA), and, in some cases, heart catheterization, to make objective assessments of coronary lesions, and according to individual needs and where necessary, to provide appropriate treatment with aspirin.

Acknowledgements Dr. Yamazaki had full access to all of the data in the study and takes responsibility for the integrity of the data and the accuracy of the data analysis. Study concept and design: Sugawara, Yamazaki, Uemura, Teramoto, Oikawa, Shimada, Uchiyama, Ando, Ishizuka, and Ikeda. Acquisition, analysis, or interpretation of data: Shimada, Uchiyama, Yamazaki, Murata, Yokoyama, and Ishizuka. Drafting of the manuscript: Sugawara, Yamazaki, Teramoto, Oikawa, Shimada, Uchiyama, and Ikeda. Critical revision of the manuscript for important intellectual content: Sugawara, Yamazaki, Teramoto, Oikawa, Shimada, Uchiyama, and Ikeda. Statistical analysis: Yamazaki, Uemura, and Ishizuka. Obtained funding: Sugawara, Goto, Shimada, Murata, Yokoyama, and Ikeda. Administrative, technical, or material support: Sugawara, Goto, Teramoto, Oikawa, and Ikeda. Study supervision: Sugawara, Yamazaki, Teramoto, Oikawa, Shimada, and Uchiyama.

JPPP Study Group information: Steering Committee: Tamio Teramoto, Kazuyuki Shimada, Shinichiro Uchiyama, Masahiro Sugawara, Yoshio Goto, Nobuhiro Yamada, Tsutomu Yamazaki, Shinichi Oikawa, and Toshiro Fujita. Data Monitoring Committee: Saichi Hosoda, Hideki Origasa, Yukito Shinohara, and Akira Yamamoto. Event Adjudication Committees: Cerebrovascular Disease Subcommittee: Shinichiro Uchiyama, Masayasu Matsumoto, and Kazuo Minematsu. Cardiovascular Disease Subcommittee: Kazuyuki Shimada, Hiroyuki Daida, and Hisao Ogawa. Other Disease Subcommittee: Kenji Yokoyama and Mitsuru Murata.

Additional contributions: We thank Takuro Shimbo, MD (Ohta Nishinouchi Hospital, Fukushima, Japan) for his advice throughout this study. Dr. Shimbo did not receive any compensation. Editorial assistance was provided by Lizzie Perdeaux, $\mathrm{PhD}$, and Jesse Alderson, $\mathrm{PhD}$ (both from Oxford PharmaGenesis Ltd., Oxford, UK), with funding from the JPPP study office.

\section{Compliance with Ethical Standards}

Conflict of interest TY reports receiving personal fees from AstraZeneca, Dainippon Sumitomo, Shionogi, and Takeda. TT reports receiving grant support from Daiichi Sankyo, Eli Lilly, Kowa, Shionogi, and Takeda; speaker fees from Amgen Astellas BioPharma, Astellas, Bayer, Daiichi Sankyo, Kissei, Kowa, Pfizer, Sanofi, and Takeda; and department endowments from Aska, Astellas, Bayer, Kissei, Kowa, and MSD. SO reports receiving personal fees from Astellas, Bayer, Daiichi Sankyo, Kyowa Hakko Kirin, Mochida, MSD, and Takeda. SU reports receiving speaker fees from AstraZeneca, Bayer, Boehringer Ingelheim, Daiichi Sankyo, Dainihon Sumitomo, Otsuka, Shionogi, Takeda, and Tanabe Mitsubishi. KA reports receiving speaker fees from Bayer, Daiichi Sankyo, Mochida, MSD, and Torii Pharmaceutical. NI reports being a former employee of Sanofi-Aventis. MM reports receiving grant support from Bayer, Daiichi Sanko, Pfizer,
Sanofi-Aventis, and Taisho-Toyama, and personal fees from DainihonSumitomo, Otsuka, and Taisho-Toyama. KY reports grants from Nippon Shinyaku, Bristol-Myers Squibb, Chugai, Takeda, Pfizer, Kyowa Hakko Kirin, and Eisai, and personal fees from Nippon Shinyaku, Celgene Japan, Bayer, Bristol-Myers Squibb, Novartis, Takeda, Pfizer, Kyowa Hakko Kirin, and Otsuka. YU reports receiving grants from the JPPP study secretariat. YI reports receiving fees for medical advice from AstraZeneca, Bayer, Daiichi Sankyo, GlaxoSmithKline, and Sanofi-Aventis. MS, YG, and KS declare no conflicts of interest.

Funding The JPPP was sponsored by the Japanese Ministry of Health, Labour, and Welfare (Grant Number 200400519A) and the Waksman Foundation of Japan. The funding sources had no role in the design and conduct of the study; collection, management, analysis, and interpretation of the data; preparation, review, or approval of the manuscript; and decision to submit the manuscript for publication. Enteric-coated 100-mg aspirin tablets were provided free of charge by Bayer Yakuhin, Japan.

Ethical approval All procedures performed in studies involving human participants were in accordance with the ethical standards of the institutional and/or national research committee and with the 1964 Helsinki declaration and its later amendments or comparable ethical standards.

Informed consent Informed consent was obtained from all individual participants included in the study.

Open Access This article is distributed under the terms of the Creative Commons Attribution-NonCommercial 4.0 International License (http://creativecommons.org/licenses/by-nc/4.0/), which permits any noncommercial use, distribution, and reproduction in any medium, provided you give appropriate credit to the original author(s) and the source, provide a link to the Creative Commons license, and indicate if changes were made.

\section{References}

1. World Health Organization. Global status report on noncommunicable diseases. 2014. http://www.who.int/nmh/publications/ ncd-status-report-2014/en/. Accessed 26 Sept 2016.

2. World Health Organization. Atlas of heart disease and stroke. 2004. http://www.who.int/cardiovascular_diseases/resources/atlas /en/. Accessed 9 Oct 2016.

3. Nippon Data Research Group. Risk assessment chart for death from cardiovascular disease based on a 19-year follow-up study of a Japanese representative population. Circ J. 2006;70:1249-55.

4. Ministry of Health Labour and Welfare Japan. Ministry of Health, Labour and Welfare Report: 2014. [Japanese]. http://www.mhlw. go.jp/wp/hakusyo/kousei/14/. Accessed 6 Oct 2016.

5. Hata J, Ninomiya T, Hirakawa Y, et al. Secular trends in cardiovascular disease and its risk factors in Japanese: half-century data from the Hisayama Study (1961-2009). Circulation. 2013;128:1198-205.

6. Nansseu JR, Noubiap JJ. Aspirin for primary prevention of cardiovascular disease. Thromb J. 2015;13:38.

7. Antithrombotic Trialists' (ATT) Collaboration, Baigent C, Blackwell L, et al. Aspirin in the primary and secondary prevention of vascular disease: collaborative meta-analysis of individual participant data from randomised trials. Lancet. 2009;373:1849-60.

8. Garcia Rodriguez LA, Martin-Perez M, Hennekens CH, Rothwell PM, Lanas A. Bleeding risk with long-term low-dose aspirin: a systematic review of observational studies. PLoS One. 2016;11:e0160046. 
9. Bibbins-Domingo K, Force USPST. Aspirin use for the primary prevention of cardiovascular disease and colorectal cancer: U.S. Preventive Services Task Force recommendation statement. Ann Intern Med. 2016;164:836-45.

10. Ikeda Y, Shimada K, Teramoto T, et al. Low-dose aspirin for primary prevention of cardiovascular events in Japanese patients 60 years or older with atherosclerotic risk factors: a randomized clinical trial. JAMA. 2014;312:2510-20.

11. Ogawa H, Nakayama M, Morimoto T, Japanese Primary Prevention of Atherosclerosis with Aspirin for Diabetes (JPAD) trial, et al. Investigators. Low-dose aspirin for primary prevention of atherosclerotic events in patients with type 2 diabetes: a randomized controlled trial. JAMA. 2008;300:2134-41.

12. Saito Y, Okada S, Ogawa H, et al. Low-dose aspirin for primary prevention of cardiovascular events in patients with type 2 diabetes mellitus: 10-year follow-up of a randomized controlled trial. Circulation. 2017;135:659-70.

13. Teramoto T, Shimada K, Uchiyama S, et al. Rationale, design, and baseline data of the Japanese Primary Prevention Project (JPPP)a randomized, open-label, controlled trial of aspirin versus no aspirin in patients with multiple risk factors for vascular events. Am Heart J. 2010;159(361-9):e364.

14. Japanese Society of Hypertension. Japanese Society of Hypertension guidelines for the management of hypertension (JSH 2004). Hypertens Res. 2006;29:S1-105.

15. Japan Atherosclerosis Society. Japan Atherosclerosis Society (JAS) guidelines for diagnosis and treatment of atherosclerotic cardiovascular diseases. 2002. http://www.researchgate.net/ publication/246586151_1FS03-4_Japan_atherosclerosis_socie ty_(JAS)_guidelines_for_diagnosis_and_treatment_of_atheroscle rotic_cardiovascular_diseases. Accessed 9 Oct 2016.

16. The Japan Diabetes Society. Evidence-based practice guideline for the treatment of diabetes in Japan [in Japanese]. Tokyo: Nankodo; 2004.

17. Roger VL, Go AS, Lloyd-Jones DM, et al. Heart disease and stroke statistics-2011 update: a report from the American Heart Association. Circulation. 2011;123:e18-209.

18. Uchiyama S, Ishizuka N, Shimada K, et al. Aspirin for stroke prevention in elderly patients with vascular risk factors: Japanese Primary Prevention Project. Stroke. 2016;47:1605-11.

19. Garcia-Rodriguez LA, Gaist D, Morton J, Cookson C, GonzalezPerez A. Antithrombotic drugs and risk of hemorrhagic stroke in the general population. Neurology. 2013;81:566-74.

20. Brotons C, Benamouzig R, Filipiak KJ, Limmroth V, Borghi C. A systematic review of aspirin in primary prevention: is it time for a new approach? Am J Cardiovasc Drugs. 2015;15:113-33.

21. Gaist D, Wallander MA, Gonzalez-Perez A, Garcia-Rodriguez LA. Incidence of hemorrhagic stroke in the general population: validation of data from the Health Improvement Network. Pharmacoepidemiol Drug Saf. 2013;22:176-82.
22. Moscucci M, Fox KA, Cannon CP, et al. Predictors of major bleeding in acute coronary syndromes: the Global Registry of Acute Coronary Events (GRACE). Eur Heart J. 2003;24:1815-23.

23. Miedema MD, Cohn JN, Garberich RF, Knickelbine T, Graham KJ, Henry TD. Underuse of cardiovascular preventive pharmacotherapy in patients presenting with ST-elevation myocardial infarction. Am Heart J. 2012;164:259-67.

24. Seshasai SR, Wijesuriya S, Sivakumaran R, et al. Effect of aspirin on vascular and nonvascular outcomes: meta-analysis of randomized controlled trials. Arch Intern Med. 2012;172:209-16.

25. Miedema MD, Duprez DA, Misialek JR, et al. Use of coronary artery calcium testing to guide aspirin utilization for primary prevention: estimates from the multi-ethnic study of atherosclerosis. Circ Cardiovasc Qual Outcomes. 2014;7:453-60.

26. Bartolucci AA, Tendera M, Howard G. Meta-analysis of multiple primary prevention trials of cardiovascular events using aspirin. Am J Cardiol. 2011;107:1796-801.

27. Raju N, Sobieraj-Teague M, Hirsh J, O’Donnell M, Eikelboom J. Effect of aspirin on mortality in the primary prevention of cardiovascular disease. Am J Med. 2011;124:621-9.

28. Fox CS, Golden SH, Anderson C, et al. Update on prevention of cardiovascular disease in adults with type 2 diabetes mellitus in light of recent evidence: a scientific statement from the American Heart Association and the American Diabetes Association. Circulation. 2015;132:691-718.

29. Meschia JF, Bushnell C, Boden-Albala B, et al. Guidelines for the primary prevention of stroke: a statement for healthcare professionals from the American Heart Association/American Stroke Association. Stroke. 2014;45:3754-832.

30. British Hypertension Society. The use of aspirin in primary prevention of cardiovascular disease. 2010. http://www.bhsoc.org/ pdfs/Statement\%20on\%20Aspirin\%20Jan10.pdf. Accessed 28 Sept 2016.

31. Mora S, Manson JE. Aspirin for primary prevention of atherosclerotic cardiovascular disease: advances in diagnosis and treatment. JAMA Intern Med. 2016;176:1195-204.

32. McNeil JJ, Wolfe R, Woods RL, et al. Effect of aspirin on cardiovascular events and bleeding in the healthy elderly. N Engl J Med. 2018;379:1509-18.

33. Li L, Geraghty OC, Mehta Z, Rothwell PM, Oxford Vascular Study. Age-specific risks, severity, time course, and outcome of bleeding on long-term antiplatelet treatment after vascular events: a population-based cohort study. Lancet. 2017;390:490-99.

34. The ASCEND Study Collaborative Group. Effect of aspirin for primary prevention in persons with diabetes mellitus. $\mathrm{N}$ Engl $\mathbf{J}$ Med. 2018;379:1529-39.

35. Gaziano JM, Brotons C, Coppolecchia R, et al. Use of aspirin to reduce risk of initial vascular events in patient at moderate risk of cardiovascular disease (ARRIVE): a randomized, double-blind, placebo-controlled trial. Lancet. 2018;392:1036-46.

\section{Affiliations}

\section{Masahiro Sugawara ${ }^{1} \cdot$ Yoshio Goto $^{2} \cdot$ Tsutomu Yamazaki $^{3} \cdot$ Tamio Teramoto $^{4} \cdot$ Shinichi Oikawa $^{5} \cdot$ Kazuyuki Shimada $^{6}$. Shinichiro Uchiyama ${ }^{7} \cdot$ Katsuyuki Ando $^{8} \cdot$ Naoki Ishizuka $^{9} \cdot$ Mitsuru Murata $^{10} \cdot$ Kenji Yokoyama $^{11} \cdot$ Yukari Uemura $^{3}$. Yasuo Ikeda ${ }^{12}$. On behalf of the Japanese Primary Prevention Project (JPPP) Study Group}

\section{Masahiro Sugawara \\ ms@sugawara.or.jp}

1 Department of Internal Medicine, Sugawara Medical Clinic, Japan Physicians Association, 3-9-16 Shukujiimachi, Nerima-ku, Tokyo, Tokyo 177-0041, Japan
2 Japan Physicians Association, Tokyo, Japan

3 Clinical Research Support Center, Center for Epidemiology and Preventive Medicine, The University of Tokyo Hospital, Tokyo, Japan 
4 Teikyo Academic Research Center, Teikyo University, Tokyo, Japan

5 Diabetes and Life Style Disease Center, Fukujyuji Hospital, Tokyo, Japan

6 Department of Cardiology, Shin-Oyama City Hospital, Tochigi, Japan

7 Clinical Research Center for Medicine, International University of Health and Welfare, Tokyo, Japan

8 Department of Internal Medicine, Kitamura Memorial Clinic, Tokyo, Japan
9 Clinical Trial Department, Cancer Institute Hospital, Tokyo, Japan

10 Clinical Laboratory, Keio University School of Medicine, Tokyo, Japan

11 Department of Hematology/Oncology, Tokai University Hachioji Hospital, Tokyo, Japan

12 Graduate School of Advanced Science and Engineering, Waseda University, Tokyo, Japan 